\title{
Le ton de Patrick Modiano : du roman ironique au roman affectif
}

\author{
Ruth Amar \\ Université de Haïfa
}

À la lecture de l'œuvre de Patrick Modiano, il semble qu'un grand changement s'est effectué dans son écriture en ce qui concerne le ton. Pierre Assouline (2003) parle d'une «musique intérieure " et considère Modiano comme un "homme de biais ». Mais à vrai dire, rien de plus difficile à décrire que le ton de Modiano. Au demeurant, qu'est-ce que le ton d'une œuvre? Question obscure, mystérieuse, essentielle cependant à l'œuvre. Selon Barthes, « [d]ans n'importe quelle forme littéraire, il y a le choix général d'un ton, d'un éthos, si l'on veut, et c'est ici précisément que l'écrivain s'individualise clairement parce que c'est ici qu'il s'engage » (1953, p. 23). 
La manière dont l'auteur se distingue est exprimée dans le ton, cette caractéristique de l'œuvre que l'on oublie souvent, qui ne s'analyse pas. Celui-ci viendrait du plus profond de soi et s'imposerait de l'intérieur, il agirait sans réflexion, il jaillirait. Là serait en somme le génie de l'écrivain : obéir à un besoin qui s'impose à lui. Au contraire, selon Derrida, "[1]e plus difficile, c'est l'invention du ton, et avec le ton, de la scène qu'on peut faire, qu'on se laisse faire, la pose qui vous prend autant que vous la prenez» (1992, p. 201). Il n'y a sans doute rien de fortuit à ce que ce soit justement la justesse du ton qui apparaisse comme «la plus difficile». Ainsi, chez Modiano, le ton échappe peut-être à l'auteur encore davantage que chez tout autre écrivain.

S'exprimant au sujet du «je», de sa relation avec la narration, Modiano évoque ce qui justement semble contribuer à la notion du ton :

Le «je» [...], c'est moi et pas moi. Mais utiliser le «Je»me concentre mieux, c'est comme si j'entendais une voix, comme si je transcrivais une voix qui me parlait et qui me disait « Je ». Ce n'est pas Jeanne d'Arc, mais plutôt comme quand on capte une voix à la radio, qui de temps en temps s'échappe, devient inaudible, et revient. Ce «Je » d'un autre qui me parle et que j'écoute me donne de la distance par rapport à l'autobiographie, même si je m'incorpore parfois au récit. (2001, p. 1)

Cette voix qui vient de loin et donne de la distance à Modiano par rapport à l'autobiographie, cette voix presque inaudible, est intimement liée au ton. Dicté par cette voix, on serait tenté de dire que, chez Modiano, le ton va l'emporter sur l'écriture. Or, ce ton est aussi influencé par la période qui le passionne le plus, à savoir la Seconde Guerre mondiale, et par la représentation de la Shoah à laquelle il se réfère sans cesse, 
surtout dans ses trois premiers romans (La Place de l'étoile, La Ronde de nuit, Les Boulevards de ceinture). Il devient clair que le ton de Modiano est intimement lié à cette question, capitale dans son œuvre: comment nommer l'innommable, saisir l'insaisissable, décrire l'indescriptible? Telle est la principale interrogation de l'écrivain, le principal défi à relever.

Le ton modianien est timbré d'une écriture paradoxale, car en examinant l'œuvre, on constate que celui-ci n'est pas homogène. Le ton du jeune écrivain des années 1970 n'est plus celui de l'écrivain mûri des années 1980. Il a subi des métamorphoses et a considérablement évolué. En comparant La Place de l'étoile (1968) à Dora Bruder (1997), transparaît tout d'abord un point commun: le sentiment de culpabilité suivi de «l'acte de vengeance » (Vardi), l'écriture; or, bien vite, on découvre que ce point commun sera traduit différemment dans les deux livres : si le ton est ironique, faisant souvent place à l'humour noir dans le premier, on se rend compte qu'il s'affaiblit pour devenir presque imperceptible dans le second, écrit quelque vingt ans plus tard, et la Shoah, omniprésente dans les deux romans, y est représentée sous des angles différents, justement grâce à l'utilisation du ton.

\section{Un ton de culpabilité}

Après la Seconde Guerre mondiale, le roman français n'est plus le même: comme le dit Alain Finkielkraut, "La Shoa est omniprésente. Ces morts ne connaissent jamais le repos.» (2000, p. 89) Or, Modiano n'a de la catastrophe aucune mémoire, ni personnelle, ni transmise. Fils d'un juif d'Alexandrie qui a échappé à l'extermination en se cachant 
derrière une double identité et des relations troubles, il n'a pas vécu la guerre. Il a néanmoins l'impression que sa mémoire précède sa naissance :

Je n'avais que vingt ans, mais ma mémoire précédait ma naissance. J'étais sûr, par exemple, d'avoir vécu dans le Paris de l'Occupation puisque je me souvenais de certains personnages de cette époque et de détails infimes et troublants, de ceux qu'aucun livre d'histoire ne mentionne. Pourtant, j'essayais de lutter contre la pesanteur qui me tirait en arrière, et rêvais de me délivrer d'une mémoire empoisonnée. J'aurais donné tout au monde pour devenir amnésique. (1977, p. 116-117)

Tous les éléments typiquement modianiens sont là : la hantise du Paris nocturne du temps de l'Occupation, la fascination par tous ces êtres mystérieux et troublants dont l'Histoire n'a même pas retenu les noms, la conscience insupportable d'être né après l'Apocalypse, le désarroi devant sa propre identité de Juif et d'enfant de la collaboration à la fois. Né en 1945, Modiano se sent personnellement coupable d'être venu au monde peu après que des millions de ses frères ont définitivement disparu dans des fours crématoires.

Dans ses romans, Modiano procède le plus souvent à la manière d'un détective ou d'un historiographe au style épuré, ce qui exige, de la part du lecteur, une complicité, une attention au moindre détail et, surtout, à ce qui est passé sous silence. Le ton de Modiano serait alors cet appel à un lecteur attentif, capable de détecter à l'oreille ce qui est dit entre les lignes : «je suis coupable».

Le fait que l'écrivain, depuis l'enfance, s'est senti à la fois victime et bourreau, juif et antisémite, français et étranger, est surtout accompagné du sentiment de culpabilité d'avoir survécu à la catastrophe, qui règnera tout au long de l'œuvre 
dès son premier livre et que l'on reconnaîtra vingt ans plus tard dans Dora Bruder : «Peut-être aurais-je voulu qu'ils se croisent, mon père et elle, en cet hiver 1942. Si différents qu'ils aient été, l'un et l'autre, on les avait classés, cet hiver-là, dans la même catégorie de réprouvés. » (p. 63) Le narrateur ressent, à travers l'identification avec Dora et son père, une affinité avec toutes les victimes de la Shoah: "Beaucoup d'amis que je n'ai pas connus ont disparu en 1945, l'année de ma naissance. » (p. 98) À travers une telle phrase, il est possible de discerner le ton de culpabilité du survivant. Modiano ne peut s'empêcher de penser que, grâce à ceux qui ont été exterminés, il peut maintenant vivre paisiblement : « D'autres, comme lui, [le père], juste avant ma naissance, avaient épuisé toutes les peines, pour nous permettre de n'éprouver que de petits chagrins. » (p. 99)

Si, dans La Place de l'étoile, il n'y a pas d'allusions directes au sentiment de culpabilité, Modiano rappelle dans Dora Bruder le fait que son premier livre est une riposte aux auteurs antisémites, découverts dans la bibliothèque de son père: "Moi, je voulais dans mon premier livre répondre à tous ces gens dont les insultes m'avaient blessé à cause de mon père. Et, sur le terrain de la prose française, leur river une fois pour toutes leur clou. » (p. 70-71) On l'aura compris, le sentiment de culpabilité a engendré chez le jeune écrivain un esprit de vengeance.

Face à la disparition, Modiano, dont l'écriture est l'ultime refuge, se met à l'œuvre. Ses textes sont imprégnés de l'événement historique dont on ne peut faire abstraction, de la souffrance liée aux conséquences de l'antisémitisme. «Écrire devient alors pour Modiano un acte de vengeance et en même temps de dévouement au père "muet devant les antisémites" et 
auquel il tient à prêter sa voix » (Vardi). Cette caractéristique est présente dans plusieurs de ses romans, ceux du début de sa carrière, mais aussi dans Dora Bruder.

\section{Une ironie résistante}

Néanmoins, après ce premier point commun entre La Place de l'étoile et Dora Bruder, apparaissent une évolution et une transformation: si les deux romans possèdent la même toile de fond, la même atmosphère, ils divergent profondément dans le ton. Ce qui contribue au ton de Modiano dans son premier roman, c'est l'utilisation de l'ironie, l'art de se moquer de quelqu'un ou de quelque chose en vue de faire réagir le lecteur. L'ironie est, chez Modiano, utilisée dans le but de dénoncer, de critiquer la réalité en termes apparemment valorisants, mais dans le but de la dévaloriser. Modiano tente de se débarrasser de ses hantises de l'Occupation par l'intermédiaire de la création d'un autre monde : celui qu'il invente à partir de sa propre interprétation de cette période. La «question juive» s'y trouve abordée sur un ton ironique: le sarcasme est censé sauver de la douleur. Modiano s'abrite derrière des propos tantôt ludiques, tantôt volontairement cyniques, et ce, pour cacher son désarroi. En fait, il se sert de la fuite dans le récit, dans la littérature, afin de ne jamais assumer entièrement la réalité des années de l'Occupation et de la Shoa dans toute leur horreur.

Ainsi, dans La Place de l'étoile, le ton de Modiano retentit comme une voix sonore, éclatante. Ces souvenirs hallucinés racontent le traumatisme psychique propre à la judaïté, la peur du juif traqué, héritage de millénaires de persécutions et de massacres, encore ravivé au $\mathrm{XX}^{\mathrm{e}}$ siècle par la tragédie de 
l'holocauste. En effet, dès l'épigraphe du livre, éclate une voix dont les échos résonnent dans tout le roman :

$\mathrm{Au}$ mois de juin 1942, un officier allemand s'avance vers un jeune homme et lui dit: "Pardon, monsieur, où se trouve la place de l'Étoile? »

Le jeune homme désigne le côté gauche de sa poitrine.

(Histoire juive.) (p. 7)

Tout au long du texte, entre les passages ironiques et les lignes noires de la tragédie du peuple juif qui est décrite en détail, la voix de Modiano se fait entendre, retentissante. Le héros narrateur, Raphaël Schlemilovitch, semble incarner tous les juifs. La voix de Modiano se confond avec celle de Schlemilovitch, assertive et émotionnelle. Comme elle, elle est vigoureuse :

Si je trouvais Léon Daudet divertissant, le colonel de La Rocque m'ennuyait. Horace de Carbuccia et Béraud m'invitaient quelquefois pour parler du complot judéo-anglais. Maurois m'enviait mes amitiés fascistes. Je lui donnais la recette: abandonner définitivement son exquise pudeur de juif honteux. Reprendre son véritable nom. Devenir comme moi, Raphaël Schlemilovitch, un juif antisémite. (p. 25)

L'ironie est ici une forme de résistance. Elle manifeste le refus de s'engouffrer dans la souffrance sans réplique. Modiano révèle la vérité violente du mal et sa manière à lui de répliquer, c'est par cette voix tonitruante du jeune écrivain qu'il est à cette période. Le 16 juillet 1942, Frédo le Gestapiste

avait fait monter Schlemilovitch père dans une traction noire : "Que dirais-tu, mon colon, d'une vérification d'identité rue Lauriston et d'un petit tour au Vel' d'Hiv'? » Puis, contemplant le costume bleu Nil de Schlemilovitch père, ses gants de chevreau violet et son foulard tango, Frédo avait ajouté : «Un vrai dandy! Vous ferez fureur à Auschwitz !» (p. 96) 
Le ton ironique, poussé à l'extrême, devient celui de l'humour noir: le passage se focalise sur le personnage de Frédo, ancien gestapiste, maintenant chauffeur de la marquise de Fougeire-Jusquiames. On se rend compte d'un mélange poignant entre bourreau et victime. Alors qu'il l'envoie à une mort certaine, Frédo se moque des vêtements ridicules du vieil homme. La marquise, sa future patronne, représente l'antisémitisme français et la collaboration. L'humour noir consiste notamment à évoquer avec détachement, et même avec amusement, les choses les plus horribles à la morale, établissant ainsi un contraste entre le caractère bouleversant ou tragique de ce dont on parle et la façon dont on en parle. Ce contraste, qui devient pour Modiano une arme de subversion, interpelle le lecteur en créant forcément chez lui une source de gêne, voire de honte et de dégoût.

Il devient clair que, dans La Place de l'étoile, Modiano aborde l'histoire d'une manière singulière, créant une atmosphère trouble où "l'écriture rejoue rhétoriquement la scène du cauchemar. Patrick Modiano compose un texte des extrêmes qui, à défaut de représenter la Shoah, laisse agir son traumatisme.» (Blanckeman, p.61) Ce climat trouble lui permet de dire ce qu'il veut, quand il veut, d'exorciser ses hantises par l'intermédiaire de la création de son propre monde, de sa propre version de la période de l'Occupation en adoptant un ton ironique, en dressant en fait une muraille littéraire entre lui-même et l'Holocauste, et ce, pour ne pas avoir à en assumer la réalité dans toute son horreur. 


\section{Un ton contre l'oubli}

Une vingtaine d'années après son premier livre, une modification s'opère dans l'écriture de Modiano. Cela est surtout dû, à mon sens, au choc ressenti à la lecture du Mémorial de la déportation des Juifs de France, publié en 1978 par Serge Klarsfeld, mais aussi peut-être à la mort du père en 1977 : le combat entre père et fils est dépassé, la volonté du fils de comprendre le père prend le dessus.

Dès sa lecture du Mémorial, Modiano commence à remettre en cause le statut de la fiction romanesque. Bouleversé par cette liste de quatre-vingts mille noms et dates de naissance, il se pose la question du rôle du roman : un récit composé selon les normes littéraires préconçues pourrait-il jamais exprimer de véritables pensées et sentiments, saisir une véritable horreur? Parlant un jour du Mémorial, il explique que ces longues listes de noms suivis d'une date et d'un lieu de naissance étaient certes le meilleur roman sur l'Occupation.

C'est alors qu'il écrit Dora Bruder. Dans ce roman, Modiano donne l'impression de tenter d'échapper à sa voix tonitruante, aux sonorités assourdissantes. Ce ton, qui est toujours le sien, se fait pourtant moins vibrant, plus timide, plus hésitant. Il n'est plus un ton de résistance, mais plutôt un ton « contre l'oubli », pour reprendre le titre d'un article d'Henri Calet (cité par Czarny) consacré aux murs de Fresnes sous l'Occupation.

Le préambule des deux romans est bien différent: si, dans La Place de l'étoile, la phrase qui ouvre le récit est très assurée — «C'était le temps où je dissipais mon héritage vénézuelien. Certains ne parlaient plus que de ma belle 
jeunesse et de mes boucles noires, d'autres m'abreuvaient d'injures. » (p. 9) —, dans Dora Bruder, l'incipit est à l'opposé : «Il y a huit ans, dans un vieux journal, Paris-Soir, qui datait du 31 décembre 1941, je suis tombé à la page trois sur une rubrique: "D'hier à aujourd'hui".» (p.7) Ici, la phrase nous plonge d'emblée dans la réalité de l'Occupation de manière réfléchie, où commence une enquête, où l'écriture de la littérature s'efface devant la prose du monde. À la première phrase qui ouvre ses récits, Modiano donne un poids incontestable. Il affirme: "C'est elle qui donne la tonalité du roman, comme les premières mesures au piano.» (2003, p. 100) À en croire Jean-Pierre Martin, le ton est «cet esprit fluide, ce génie ou ce démon qui surgit dès la première phrase, ne quitte pas le livre, jusqu'à son dernier mot» (p. 264) C'est très exactement le cas des romans de Modiano. Et en ce qui concerne Dora Bruder, un autre ton est donné, une autre voix s'impose au lecteur, un autre cérémonial linguistique.

En effet, dans ce roman publié en 1997, Modiano interroge la mémoire tout en intégrant les thèmes de la Seconde Guerre mondiale et de l'Holocauste. Mais cette fois, on ressent la fragilité du héros narrateur, aussi bien d'ailleurs que celle des autres personnages. Au fil de son enquête, le lecteur partage ses doutes, son impression de vide et d'absence chaque fois qu'il évoque le destin de la famille Bruder. L'identité de ses membres les a conduits à la mort alors que ceux qui ont survécu sont marqués pour Modiano d'un autre sceau. « Nous sommes condamnés à être des collabos» (2007, p. 51), affirme-il. Il crée un climat lourd, angoissant, où s'installe une voix chancelante. Il incarne le passé douloureux dans des personnages vulnérables, fragiles, qui osent à peine surgir 
du brouillard, ce qui produit un effet de disharmonie et contribue à la construction de cette nouvelle voix.

\section{L'humilité d'une histoire simple}

Le ton de Dora Bruder est plus fragile. Il se fait plus délicat, plus discret. À l'occasion de la parution de son avant-dernier livre, Un pedigree, Modiano déclare :

Je ne peux pas trop employer dans la fiction cet «humour discret, plutôt noir et décapant », parce que, à trop forte dose, cela orienterait la fiction vers la satire, et j'ai besoin que les personnages de fiction me fassent rêver. (2005)

Du coup, on s'aperçoit que, depuis déjà une dizaine d'années, le ton de Modiano n'est plus ironique, mais plutôt tendre et modéré. La «musique intérieure »s'infiltre dans le texte presque de manière silencieuse. «Au cinéma on peut chuchoter, comme dans un roman » (1997b), affirme-t-il. Plus aucune trace d'ironie. Dans Dora Bruder, on est aux antipodes de l'humour noir. Le père n'est plus nommé Schlemilovitch; il n'est plus arrêté par Frédo le Gestapiste, mais tout simplement par des administrateurs français collaborateurs qui obéissent aux nouvelles lois de l'Occupation. Les bourreaux sont nommés sans ambiguïté. Ainsi, le commissaire Jacques Schweblin, de la Police des Questions Juives, est évoqué. Modiano rappelle l'horreur et l'absurdité de la disparition du peuple juif de Paris avec tout le sérieux qui s'impose devant la tragédie du peuple juif :

On avait imposé des étoiles jaunes à des enfants aux noms polonais, russes, roumains, et qui étaient si parisiens qu'ils se confondaient avec les façades des immeubles, les trottoirs, les infinies nuances de gris qui n'existent qu'à Paris. Comme Dora 
Bruder, ils parlaient tous avec l'accent de Paris, en employant des mots d'argot dont Jean Genêt avait senti la tendresse attristée. (p. 138-139)

Le désir de prouver son habileté d'écrivain et sa performance artistique cède la place à l'humilité d'une histoire simple, réelle et cruelle, au témoignage émouvant de la disparition d'une adolescente juive. Dora Bruder est le nom d'une véritable adolescente qui figure dans les listes de départ vers Auschwitz, le 18 septembre 1942, et dans le Mémorial de Klarsfeld. Il s'agit au départ plus d'une enquête que d'un roman. Cette enquête commence en 1988 quand l'écrivain, s'apprêtant à concevoir son récit, consulte les vieux journaux et annuaires d'où naissent ses rêveries romanesques. Les procédés si chers à l'auteur de Livret de famille ou d'Un cirque passe sont employés pour résoudre l'énigme d'une existence réelle. Modiano part d'un article de presse, mène des investigations dans Paris, en quête des rares papiers officiels, observe des traces sur les façades, recherche à travers les immeubles modernes, le souvenir des bâtiments détruits, erre dans des périphéries qui ont perdu toute mémoire de celles et de ceux qui les peuplèrent :

Je me suis dit que plus personne ne se souvenait de rien. Derrière le mur s'étendait un no man's land, une zone de vide et d'oubli. Les vieux bâtiments des Tourelles n'avaient pas été détruits comme le pensionnat de la rue de Picpus, mais cela revenait au même.

Et pourtant, sous cette couche épaisse d'amnésie, on sentait bien quelque chose, de temps en temps, un écho lointain, étouffé, mais on n'aurait été incapable de dire quoi, précisément. C'était comme de se trouver au bord d'un champ magnétique, sans pendule pour en capter les ondes. (p. 131)

L'écriture est essentiellement dépouillée. Modiano énumère des faits et constate une étrange coïncidence entre le 
destin de la jeune juive déportée et celui de son père et, pardelà celui-ci, entre tous les êtres traqués de l'Occupation. Le Modiano mûri des années 1990 a retiré le masque du bouffon pour aborder son sujet avec toute la sensibilité qu'il mérite. Dora Bruder est un récit fondé sur le fait divers d'une très jeune fille traquée et rompue par la machinerie de l'Occupation. L'histoire d'une pauvre adolescente en fuite que le narrateur tente à tout prix de faire revivre afin de la soutirer de l'oubli. Modiano parcourt ainsi le Paris des années 1990 dans l'espoir de ressusciter le fantôme de la mystérieuse jeune fille qui hante ses rues. Bien davantage qu'à un roman traditionnel, son texte fait penser à une véritable topographie de la souffrance et de la mort. Le ton est alors celui de l'émoi; presque imperceptible, il se fait plus tendre.

\section{Entre les mots laconiques : le chuchotement}

Le ton est aussi intimement relié au rythme. Le rythme de Dora Bruder fluctue de manière ambiguë entre des chapitres courts, rapides (comme celui des pages 112-113, constitué du registre des femmes internées aux Tourelles), et des chapitres plus chargés. Modiano, artiste de l'ellipse et de la concision, prend une nouvelle voie (voix). Le récit procède alors par notations. Il a une préférence marquée pour la parataxe et une juxtaposition des détails : "Lever vers six heures. Chapelle. Salle de classe. Réfectoire. Salle de classe. Cour de récréation. Réfectoire. Salle de classe. Étude du soir. Chapelle. Dortoir. Sorties, les dimanches. » (p. 39-40)

Ton dissimulé, semble-t-il, qui cache quelque chose, glissé entre les mots laconiques. Ton sans identité, phrases où 
s'effacent le sujet et le verbe, qui cherchent à produire une sensation forte. Jeu entre fiction et réalité, entre roman et vie. Ces mots secs et dénudés/dénués de tout sentiment viennent renforcer la description glaciale de ce qui a pu être le quotidien de Dora, attirant l'attention sur le fait que sa vie entre les murs de l'internat avait dû être bien froide et marquée par une profonde solitude. Le narrateur conclut d'ailleurs par ce seul mot qui s'impose et vient tout résumer : «Solitude » (p. 41). Derrière ces lignes dépouillées se cache cette fois la voix muette de l'auteur, celle qui voudrait dire une souffrance insoutenable, mais qui ne fait que la montrer du doigt. D'ailleurs, Modiano s'exprimait déjà sur ce point en 1981 : «Je n'ai aucune facilité de plume, et écrire est donc pour moi un travail un peu pénible, bien que le résultat donne une impression de simplicité. J'essaie de dire les choses avec le moins de mots possible. » (1981, p. 56-57)

Les textes de Dora Bruder présentent des caractéristiques du style coupé. Ainsi, la trame resserrée du début du roman est celle d'un avis de recherche lu dans une petite annonce de Paris-Soir : " On recherche une jeune fille, Dora Bruder, 15 ans, $1 \mathrm{~m} \mathrm{55,} \mathrm{visage} \mathrm{ovale,} \mathrm{yeux} \mathrm{gris-marron,} \mathrm{manteau} \mathrm{sport} \mathrm{gris,}$ pull-over bordeaux, jupe et chapeau bleu marine, chaussures sport marron.» (p.7) Par la suite, le récit ne fera que commenter cet avis à chaque chapitre sans véritablement progresser dans les recherches, car toutes les tentatives pour reconstituer le parcours de la jeune fille échouent: «On se dit qu'au moins les lieux gardent une légère empreinte des personnes qui les ont habités. Empreinte : marque en creux ou en relief. Pour Ernest et Cécile Bruder, pour Dora, je dirai : en creux. J'ai ressenti une impression d'absence et de vide, chaque fois que je me suis trouvé dans un endroit où ils avaient vécu. » (p. 28-29) 
Le roman se termine sur le fait que Dora Bruder emporte son secret avec elle et que personne ne saura jamais comment elle a passé ces journées de fugue avant sa déportation :

J'ignorerai toujours à quoi elle passait ses journées, où elle se cachait, en compagnie de qui elle se trouvait pendant les mois d'hiver de sa première fugue et au cours des quelques semaines de printemps où elle s'est échappée à nouveau. C'est là son secret. Un pauvre et précieux secret que les bourreaux, les ordonnances, les autorités dites d'occupation, le Dépôt, les casernes, les camps, l'Histoire, le temps - tout ce qui vous souille et vous détruit — n'auront pas pu lui voler. (p. 144-145)

Dans cette voix chuchotée de Modiano, s'inscrivent de nombreux signes d'oralité. Ainsi, toutes les expressions hésitantes, toutes les formulations en forme de litanies qui tempèrent le récit, par lesquelles la narration s'adresse au lecteur et donne à l'écriture la signification d'un geste. On y entend tel un refrain incontrôlable des mots répétés: "Je ne savais encore rien » (p. 29) et « je ne sais pas » (p. 87, 90, 96, 125), « on ne saura jamais» (p. 76, 108), « je savais vaguement » (p. 100), « Je me demande» (p. 38, 73, 109, 123), « on se demande» (p. 92), « je me demandais si » (p.61), « Je suppose » (p. 25, 40, 55, 83), «Cela suppose» (p. 109 ?), «Qui sait? » (p. 59) «Longtemps je n'ai rien su de Dora Bruder» (p. 60), « je doute » (p. 57), «j'en doute » (p. 102), « j'ignore si » (p. 74, 83, 127), «j'ignorerai toujours » (p. 144). Bref, une voix refoulée, pleine du regret de ne pouvoir élucider l'histoire de Dora Bruder.

Ces expressions sont renforcées par des modalisations comme « sans doute » (p. 90), « peut-être » (p. 40, 63, 110, 141) et par des conditionnels. De tels effets de répétition, qui sont une sorte de mimesis du parlé dans l'écrit, transcrivent des 
marques d'affectivité et renvoient à une identification sensible, absente dans les autres romans (comme si Modiano se l'était interdit auparavant), comme s'il se livrait de manière ostentatoire dans Dora Bruder, peut-être pour donner libre cours à cette voix émotive refoulée jusqu'ici. Cette voix brisée, à peine perceptible, est la voix d'un manque, d'une hésitation, d'une quête infinie qui relèverait aussi de l'oralité qui est, selon Jean-Pierre Martin, «cette impulsion respiratoire, ce souffle vital, cette présence d'un corps énonciateur et proférateur qui peut traverser l'écrit comme le parlé » (p. 263). Cette nouvelle voix modianienne s'accompagne de nombreuses questions sans réponses qui scandent le texte et lui donnent un rythme étonnant. Elles viennent briser l'enquête sèche du polar et transforme l'histoire de Dora Bruder en un récit poétique affectif et émouvant. Comme le mentionne Blanckeman, ce récit constitue

un geste de vigilance face à toute exaction possible de l'histoire, un acte de résistance à toute forme d'amnésie et une ode discrète mais soulignée au pouvoir de la littérature, pour peu qu'elle admette ses propre limites et respecte le secret des morts quand elle en transmet le souvenir (2009, p.134).

Du roman ironique au roman affectif, comme nous avons pu le constater, le ton de Modiano diverge entre La Place de l'étoile et Dora Bruder. Certes, dans les deux cas, il émerge des tréfonds du décor lourd de la Shoah et cherche dire ce qu'il est impossible de dire. Mais alors que La Place de l'étoile est un roman de colère, Dora Bruder est dicté par un ton qui se veut délicat et qui laisse transparaître les émotions de l'auteur. 


\section{Bibliographie}

ASSOULINE, Pierre (2003). « Les Carnets », Lire, no 319, oct., p. 7. BARTHeS, Roland (1953). Le Degré zéro de l'écriture, Paris, Seuil. Blanckeman, Bruno (2009). Lire Patrick Modiano, Paris, Armand Colin, coll. "Lire et comprendre. Écrivains au présent ».

CZARNY, Norbert (1997). "Contre l'oubli», La Quinzaine littéraire, no 714, 16 avril, p. 7-8.

DERRIDA, Jacques (1992). « Heidegger, l'enfer des philosophes » [entretien avec Didier Éribon, Le Nouvel Observateur, 612 nov. 1987], dans Points de suspension, Paris, Galilée, p. 193-202.

FINKIELKRAUT, Alain (2000). Une voix vient de l'autre rive, Paris, Gallimard.

Gaudemar (de), Antoine (2001). "Modiano, souvenir écrin », Libération, 26 avril, p. 1-2.

KLARSFELD, Serge (1978). Le Mémorial de la déportation des Juifs de France : listes alphabétiques par convois des Juifs déportés de France, historique des convois de déportation, statistiques de la déportation des Juifs de France, Paris, Association pour le jugement des criminels nazis qui ont opéré en France.

MARTIN, Jean-Pierre (1998). La Bande sonore. Essai sur le roman de la voix, Paris, José Corti.

Modiano, Patrick (1968). La Place de l'étoile, Paris, Gallimard.

- (1977). Livret de famille, Paris, Gallimard.

— (1981). «Entretien », Paris Match, 13 mars, p. 56-57.

- (1997a [1999]). Dora Bruder, Paris, Gallimard, coll. « Folio ». 
- (1997b). «Cannes et autres lieux de mémoire: Catherine Deneuve et Patrick Modiano", entretien avec Frédéric Bonnaud, Les Inrockuptibles, no 103, 7-13 mai, p. 20-29.

- (2001). "Modiano, souvenir écrin », entretien avec Antoine de Gaudemar (de), Libération, 26 avril, p. 1-2.

- (2003). « Modiano », entretien avec Laurence Liban, Lire, no 319, p. 98-104,

<http://www.lexpress.fr/culture/livre/modiano 808386.html>.

- (2005). «Rencontre avec Patrick Modiano, à l'occasion de la parution d'Un Pedigree »,

<http://www.gallimard.fr/catalog/Entretiens/01052120.htm>.

- (2007). Cité par Aliette Armel, «Enfants de victimes et de bourreaux », Le Magazine Littéraire, no 467, 1 1er sept., p. 50-54.

VARDI, Charlotte (2007). "Patrick Modiano et George Perec », conférence, Centre culturel français de Haïfa, mars.

\section{Résumé}

Cette étude analyse la manière dont s'exprime le ton de Modiano, la manière par laquelle l'auteur s'individualise. Un ton difficile à saisir, car s'il paraît au premier abord neutre, derrière les phrases, se cachent des éléments qui contribuent à un ton spécifique, voire à la voix de l'auteur. Au fil de l'œuvre, l'on découvre que la voix de Modiano n'est pas homogène. Le ton du jeune écrivain des années 1970 n'est pas celui des années 1980. Il s'est métamorphosé et a évolué de manière considérable. En comparant La Place de l'étoile et Dora Bruder, on est conscient de la transformation : le ton retentissant des débuts de Modiano 
s'est affaiblit, devenant presque imperceptible, reflétant le fait que la représentation de l'holocauste dans ces deux livres est bien différente.

\begin{abstract}
In this paper I will explore the way in which the characteristics of Modiano's tone are expressed in the texts, the way in which the author individualizes himself. A tone so hard to cease as it seems at first neutral, but behind the sentences, are hidden elements that contribute to the specific tone and even to the voice of the writer. In considering the work, one finds that the voice of Modiano is not homogeneous. The tone of young writer of the seventies is no longer that of the writer of the nineties. He has suffered metamorphosis and has evolved significantly. In comparing La Place de l'étoile to Dora Bruder, one becomes well aware of the transformation: the tone first shining and thundering, is weakened to become, almost imperceptible and reflects the fact that the representation of the Holocaust in these two books is quite different.
\end{abstract}

